\title{
Interaction between mitral valve apparatus and left ventricle. Functional mitral regurgitation: A brief state-of-the-art overview
}

\author{
Erwan Donal ${ }^{A-\mathrm{F}}$, Vasileios Panis ${ }^{\mathrm{A}-\mathrm{F}}$ \\ Rennes University Hospital, University of Rennes, France \\ A - research concept and design; $\mathrm{B}$ - collection and/or assembly of data; $\mathrm{C}$ - data analysis and interpretation; \\ $D$ - writing the article; $E$ - critical revision of the article; $F$ - final approval of the article
}

Address for correspondence

Erwan Donal

E-mail: erwan.dona@@chu-rennes.fr

Funding sources

None declared

Conflict of interest

None declared

Acknowledgements

The authors would like to gratefully acknowledge

the contribution made by Professor Wojciech Kosmala.

\begin{abstract}
In this overview, we described the mitral valve anatomy, focusing on its anatomical and functional relationships with the left ventricle (LV), and how an impaired coordination between the two can lead to valvular dysfunction with serious clinical consequences. In the $1^{\text {st }}$ part of this overview, we sought to describe the anatomy of the mitral valve apparatus. In the $2^{\text {nd }}$ part, we sought to analyze the interactions of the LV with the mitral valve, the possible etiologies that cause mitral regurgitation (MR), and therapeutic strategies that can be utilized nowadays in the effort to reinstate normal valvular function. The comprehension of these mechanisms makes it possible to implement appropriate therapeutic solutions in order to alleviate the burden of mitral valve disease.
\end{abstract}

Key words: treatment, anatomy, restriction, mitral regurgitation, atrial

Received on August 7, 2021

Reviewed on September 12, 2021

Accepted on October 25, 2021

Published online on October 27, 2021

Cite as

Donal E, Panis V. Interaction between mitral valve apparatus and left ventricle. Functional mitral regurgitation: A brief state-of-the-art overview. Adv Clin Exp Med. 2021;30(10):991-997.

doi:10.17219/acem/143324

DOI

10.17219/acem/143324

Copyright

Copyright by Author(s)

This is an article distributed under the terms of the

Creative Commons Attribution 3.0 Unported (CC BY 3.0)

(https://creativecommons.org/licenses/by/3.0/) 


\section{Introduction}

The mitral valve has a dual role: to facilitate the flow from the left atrium (LA) to the left ventricle (LV) during diastole, and to interact with the LV and protect the LA from blood reflux during systole. The mitral valve is the last barrier between the low pressure system of the LA and pulmonary arteries, and the high pressure system of the LV and the aorta, which means that it withstands one of the greatest forces in terms of pressure difference in the human body. ${ }^{1}$ In the $1^{\text {st }}$ part of this overview, we sought to describe the anatomy of the mitral valve apparatus. In the $2^{\text {nd }}$ part, we sought to analyze the interactions of the LV with the mitral valve, the possible etiologies that cause mitral regurgitation (MR), and therapeutic strategies that can be utilized nowadays in an effort to reinstate normal valvular function.

\section{Mitral valve apparatus}

The normal mitral valve is definitely one of the most complex anatomical structures as it consists of 6 main components: the mitral annulus (MA), the anterior (AL) and posterior (PL) leaflet, the chordae tendineae, the papillary muscles (PM), and the LV (Fig. 1). A precise temporal and spatial motion of each of the components results in the unobstructed and unidirectional blood flow into the LV. Any abnormalities in this sequence of events can create a serious malfunction. Its clinical manifestations are described as mitral stenosis (MS) when there is obstruction during diastole, MR when there is blood backflow during systole, or mixed valve disease when the 2 situations combine.

\section{Mitral annulus}

The MA shape is described as a "saddleback" with a hyperbolic paraboloid oval geometry. It lies in the intersection between LA and LV, over the mitral leaflets. The MA has nerves and it supplies the mitral leaflets with blood vessels. ${ }^{1}$ The annular orifice area has normally $4-6 \mathrm{~cm}^{2}$. The anterior section, which is connected with aortomitral continuity, constitutes the atrial part of the saddle and is more stable during the cardiac circle. The posterior section includes the lowest part of the saddle, closer to the lateral and medial commissures, and it can move more freely. ${ }^{2}$ During diastole, the MA stays relatively unchanged, but during systole, the MA changes its shape: it deepens the saddle and shortens the anteroposterior diameter, resulting in an overall $25 \%$ reduction of the mitral valve area. ${ }^{3}$

\section{Mitral leaflets}

The most essential part of the valve are the mitral leaflets. The anterior mitral valve leaflet (AML) has a shape of a sail, is located anteriorly in close proximity to the noncoronary cusp (NC), and covers $2 / 3$ of the perimeter of MA. The AML is larger, thicker and stronger than the PL, and although not anatomically separated, it is divided into 3 scallops: lateral A1, central A2 and medial A3. The posterior or mural leaflet has a crescentic shape, covers $2 / 3$ of the MA and has 3 anatomically distinct scallops: lateral P1, central P2 and medial P3, opposed to the anterior counterparts. During systole, the leaflets approach, attach and form a single semilunar coaptation line. Their ends form the anterolateral and posteromedial commissure, respectively to the underlying PM. ${ }^{4,5}$ Nevertheless, the mitral leaflets are not composed of so-called "dead" tissue. In pathological situations with increased mechanical stress, there

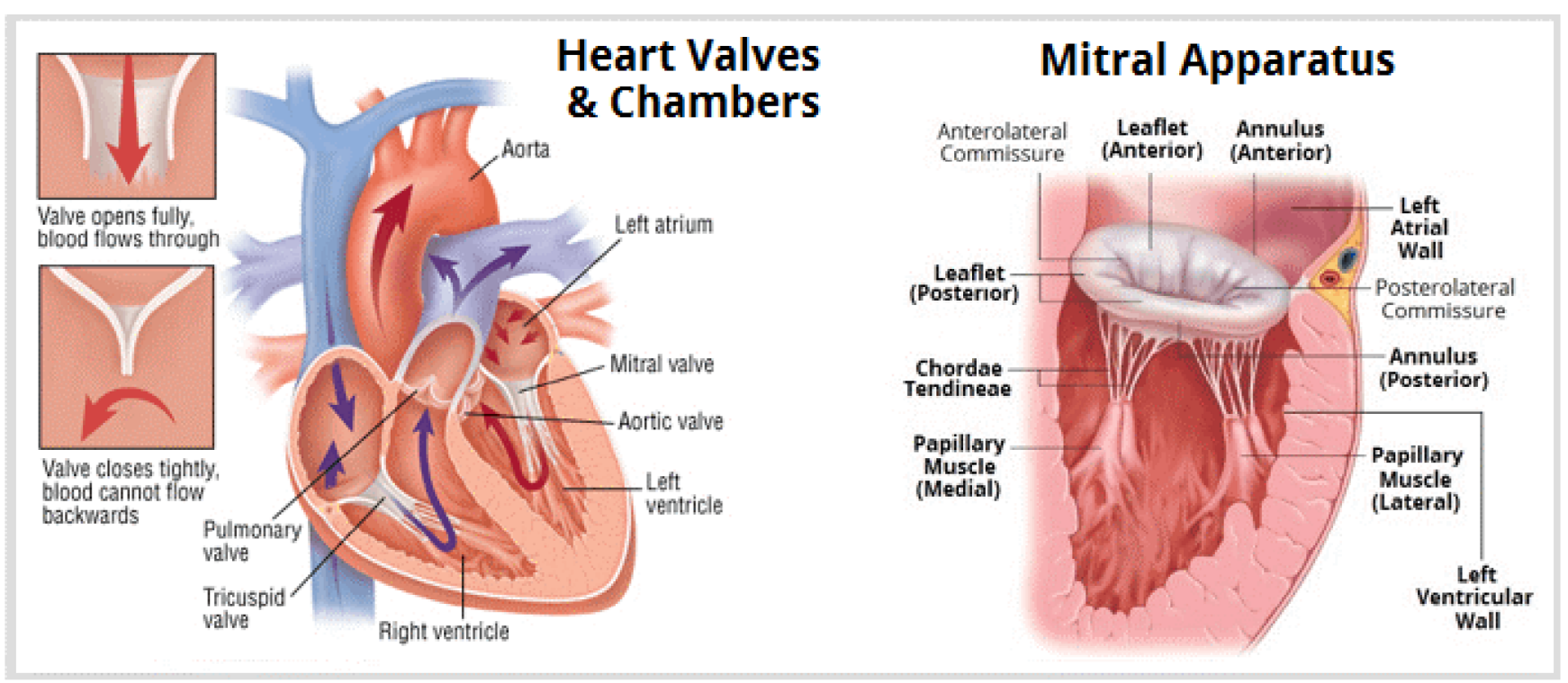

Fig. 1. Basic anatomy of heart valves and chambers (mitral valve apparatus) 
is a significant increase in both valve surface and thickness through the transdifferentiation from endothelial to mesenchymal cells and increased collagen deposition., ${ }^{4,5}$

\section{Papillary muscles and chordae tendineae}

The subvalvular apparatus is composed of the 2 PM named after the concordant commissures, and the chordae tendineae. The base of the PM is attached to the apical 1/3 of the ventricle. The anterolateral PM is single-headed and has a dual blood supply. On the other hand, the posteromedial PM is usually double-headed and has a single blood supply, which makes it more susceptible to ischemia. ${ }^{6}$ From the tips of the PM, numerous chordae tendineae with various patterns arise and attach to the ventricular side of the mitral leaflets. ${ }^{7}$ There are 2 types of chords: a) the primary chords, which are thinner, less elastic and attached to the free edge of the leaflets; they protect the leaflets from inversion and flail into the atrium during systole; b) the secondary chords, which are thicker and more extensible; they are anchored to the anterior rough zone and the PL main body, as they stabilize the subvalvular geometry. ${ }^{7}$ The importance of PM anatomy is more apparent in patients with hypertrophic cardiomyopathy (HCM), where specific abnormalities such as PM hypertrophy, bifid mobility and anteroapical displacement of the anterolateral PM have been well described in both echocardiography and cardiovascular magnetic resonance imaging (CMR) studies. These variations can provoke an increased left ventricular outflow tract (LVOT) obstruction with systolic anterior motion (SAM), while the shifting of the AML towards the septum makes the valve more susceptible to malcoaptation and regurgitation. ${ }^{8}$ Finally, in some cases of HCM, there is a direct attachment of PM head to AML without chords. It leads to a long mid-cavity narrowing with LVOT obstruction without SAM. ${ }^{7}$

\section{Interaction with the left ventricle}

The LV anatomy and function are quite important for the proper valve function. During the early systole, the PM base and tips move along the longitudinal contraction towards the atrium, following the upward motion of the leaflets. At the same time, the MA contracts and folds along the intercommissural line, allowing the effective early coaptation of the leaflets and saddle shape accentuation. In the midsystole and late systole, the closing forces created by the increased gradient between LV and LA promote the sealing of the valve, while the tethering forces transmitted by the LV-wall PM chords to the leaflets keep the coaptation point in the annular plane, preventing prolapse or flail., ${ }^{9,10}$ Simultaneously, the PM contract and reduce their length, keeping both leaflets under direct tension and restrain, thus preventing systolic anterior motion and obstruction of the LVOT. The properly timed activation of the LV and the simultaneous contraction of the PM ensure the homogenous movement of the subvalvular mechanism, and the application of symmetrical tethering forces to the mitral leaflets throughout the systolic period. Even mild alterations in this interplay among the main components of the mitral valve complex can unbalance the closing and tethering forces, creating a deficit in the coaptation line, with the occurrence of MR.

\section{Mitral regurgitation classification}

The original description of the mechanisms of MR based on the leaflet mobility was provided by Alain Carpentier (Fig. 2). ${ }^{11}$ More analytically, in type I MR, there is a normal leaflet motion and the MR is the result of the annular dilation or perforation of the leaflet; typical causes include annular dilation with LA enlargement due to the chronic

\section{Mitral Regurgitation}

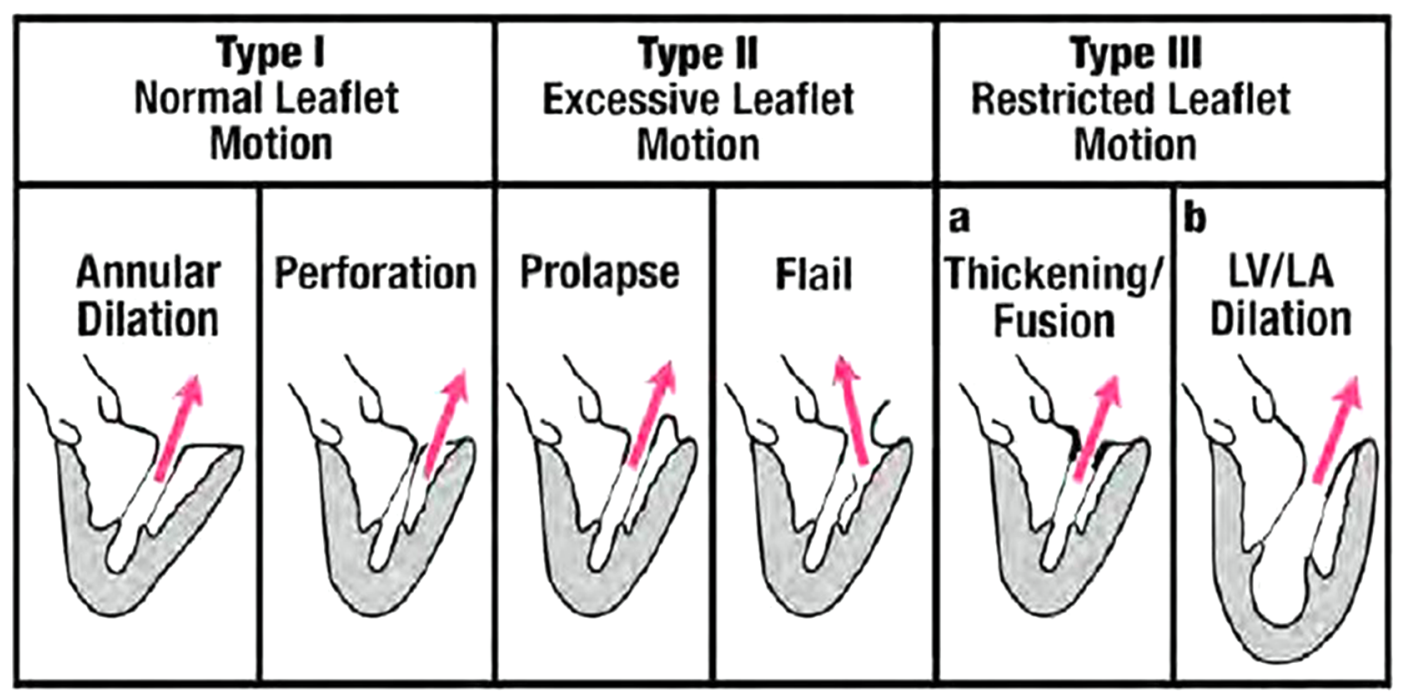


atrial fibrillation for the former, and infectious endocarditis for the latter. In type II, there is an excessive leaflet motion, where MR is provoked by prolapse or ruptured chordae and classical etiologies include fibroelastic deficiency (FED) and myxomatous degeneration (Barlow's disease).

In type III, there is a restricted leaflet motion, and more specifically, in type IIIa, there is a systolic and diastolic restriction caused by inflammation, rheumatoid valve disease or carcinoid disease. In type IIIb, there is only systolic restriction due to the LV dilation, PM displacement and increased tethering forces applied on the mitral leaflets. From these categories, type I with annular dilation, type II with prolapse and type IIIb are more suitable subtypes for the newest transcutaneous therapies when the risk of surgical intervention is too high. ${ }^{12}$

\section{Functional mitral regurgitation}

Functional mitral regurgitation (FMR) occurs due to the LV dysfunction despite apparently normal leaflet anatomy and motion, and is very frequently a consequence of ischemic heart disease or dilated cardiomyopathy. This is the "classical" secondary MR that falls into IIIb category of Carpentier classification. Of note, the annulus is usually enlarged when the ventricle is sick, but it is believed that IIIb type, not I, is the predominant mechanism in this category.

Type IIIb MR is undoubtedly caused by LV remodeling and it is attributed to the global LV enlargement, increased LV sphericity, MA enlargement, and apical/posterior/posterolateral and upward displacement of PM. ${ }^{13}$ Moreover, there is an altered annular geometry with an increased anteroposterior diameter, loss of annular folding and the absence of the saddle-shaped accentuation that is more obvious in early systole. Concerning the PM

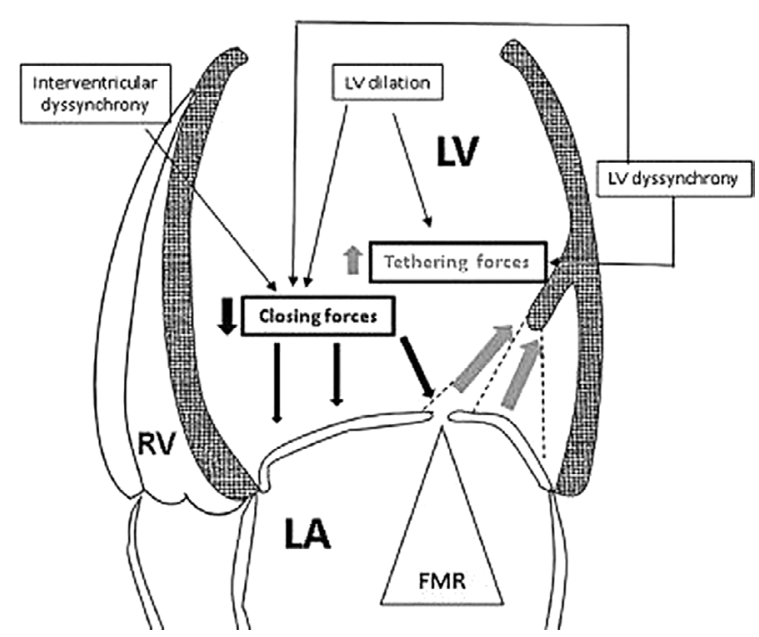

Fig. 3. Schematic pathophysiology in type IIIb mitral regurgitation (MR) - four-chamber (4Ch) view of left ventricle (LV) with the functional mitral regurgitation (FMR)

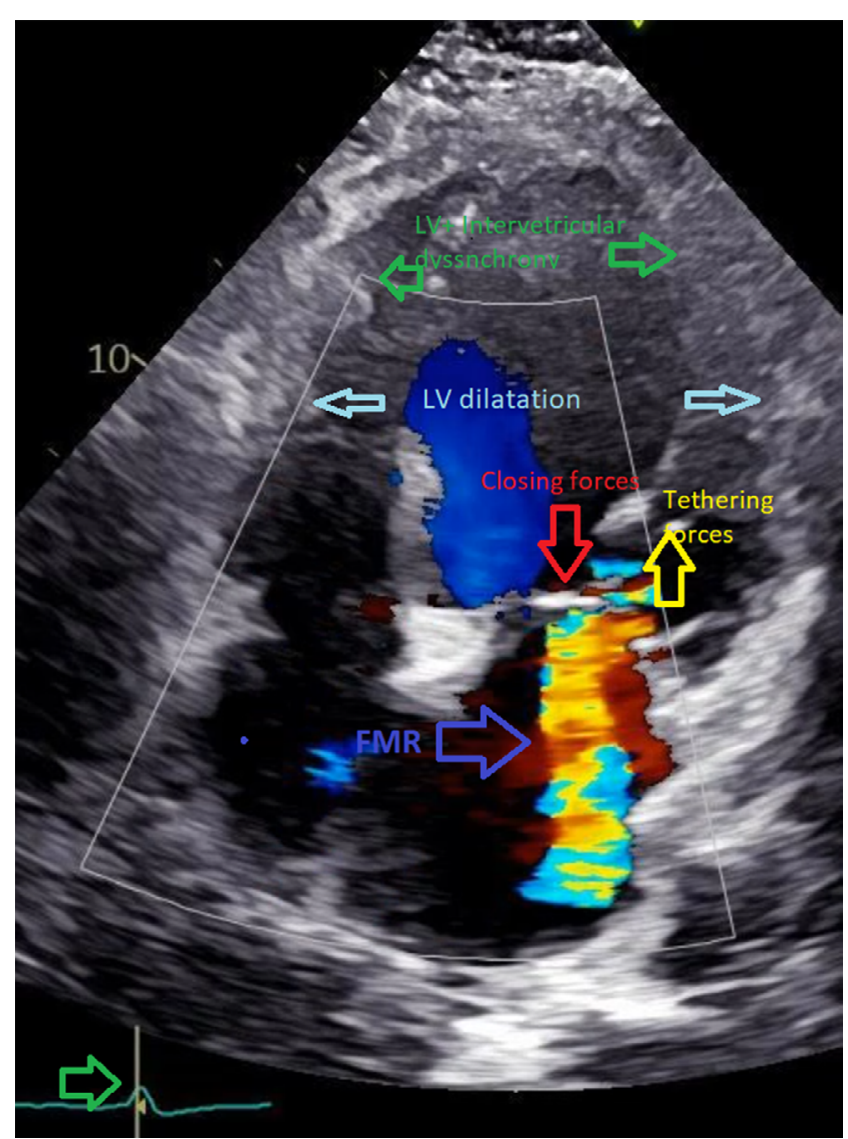

Fig. 4. Main characteristics of Mitra-FR and COAPT studies. Note the differences in effective regurgitant orifice area (EROA) and left ventricle enddiastole volume (LVEDV) which led to the differentiation of proportionate from disproportionate functional mitral regurgitation (FMR)

in FMR, there is an increased tip to annulus distance, decreased PM attenuation during midsystole, paradoxical relocation of the PML, and asymmetric descent towards the annulus. As a result, there is an increased tethering of the mitral leaflets, excessive tenting and apical displacement of the coaptation line, all of which lead to ineffective sealing of the mitral valve and MR (Fig. 3,4). ${ }^{13}$

\section{Atrial functional mitral regurgitation}

A different entity of functional MR with unique characteristics and pathophysiology, secondary to annular dilation due to the atrial enlargement, is referred to as atrial functional MR (AFMR). ${ }^{14}$ In the original description by Carpentier, type I MR (normal leaflet motion) included almost exclusively organic disease. Yet, in the current era, this can be completely reversed due to the exponential increase in prevalence of atrial fibrillation, mainly in the older population. The AFMR occurs in the context of $\mathrm{AF}$ and/or heart failure (HF) with preserved ejection fraction (HFpEF), and its anatomic culprits are annular dilation, the absence of leaflet growth and impaired annular dynamics. The annular dilation usually does not cause 
Table 1. The differences between MITRA-FR and COAPT trials

\begin{tabular}{|c|c|c|}
\hline MITRA-FR compared to COAPT & MITRA-FR (304 pts at 37 centers) & COAPT (610 pts at 89 centers) \\
\hline Technical implantation success & $96 \%$ & $98 \%$ \\
\hline EROA (mean $\pm S D)$ & $31 \pm 10 \mathrm{~mm}^{2}$ & $41 \pm 15 \mathrm{~mm}^{2}$ \\
\hline LVEDV (mean \pm SD) & $135 \pm 35 \mathrm{~mL} / \mathrm{m}^{2}$ & $101 \pm 34 \mathrm{~mL} / \mathrm{m}^{2}$ \\
\hline GDMT at baseline and FU & $\begin{array}{l}\text { variable adjustment in each group per } \\
\text { "real-world" practice }\end{array}$ & $\begin{array}{c}\text { GDMT at baseline, } \\
\text { few major changes during FU }\end{array}$ \\
\hline Different primary endpoints & $\begin{aligned} & 54.6 \% / 51.3 \% \\
p= & \text { NS at } 12 \text { months }\end{aligned}$ & $\begin{array}{l}151 \text { of } 283 / 92 \text { of } 160 \text { at } 24 \text { months } \\
\qquad p<0.001\end{array}$ \\
\hline Mortality at 1 year & $\begin{array}{l}\sim 23 \% \\
p=N S\end{array}$ & $\begin{array}{l}28 \% \text { compared to } 22 \% \\
\qquad p<0.001\end{array}$ \\
\hline $\mathrm{MR} \geq 2$ at $\mathrm{BS} \boldsymbol{\rightarrow} 12$ months $\boldsymbol{\rightarrow}>24$ months & $8 \% \rightarrow 17 \% \rightarrow ?$ & $7.4 \% \rightarrow 5.3 \% \rightarrow 0.9 \%$ \\
\hline
\end{tabular}

EROA - effective regurgitant orifice area; SD - standard deviation; BS - baseline; LVEDV - left ventricle end-diastolic volume; GDMT - guideline-directed medical therapy; FU - follow-up; NS - non-significant; COAPT - Clinical Outcomes Assessment of the MitraClip Percutaneous Therapy for Extremely High Surgical Risk Patients; MITRA-FR - Multicentre Randomized Study of Percutaneous Mitral Valve Repair MitraClip Device in Patients With Severe Secondary Mitral Regurgitation.

severe MR because of the intact nature of the subvalvular mechanism and its ability to counterbalance the coaptation deficit. However, as annular dilation progresses, the protective mechanism may be lost. This can lead to severe functional MR, with no restriction or tethering of the leaflets. ${ }^{15}$ In patients with such condition, maintaining sinus rhythm may be of great value. Gertz et al. had shown that after a successful AF ablation and no recurrences of AF, there was a significant reduction in LA and MA sizes, and only $1 / 3$ of patients had severe residual MR. ${ }^{16}$

\section{Proportionate and disproportionate secondary mitral regurgitation}

Macro- and microalterations in the LV-MV relationships became more obvious in the post hoc analysis of 2 important trials of the last decade - Multicentre Randomized Study of Percutaneous Mitral Valve Repair MitraClip Device in Patients With Severe Secondary Mitral Regurgitation (MITRA-FR) and Clinical Outcomes Assessment of the MitraClip Percutaneous Therapy for Extremely High Surgical Risk Patients (COAPT) - that evaluated transcatheter mitral valve replacement (TMVR) in functional MR and provided us with a new pathophysiological concept for understanding functional MR. ${ }^{17-20}$ In order to coalesce the contradictory results of the 2 trials, a new conceptual framework is proposed with 2 subtypes of FMR:

1. As "proportionate" is described the MR whose severity is predicted and explained by the LV dilation and symmetrical tethering of MV leaflets mimicking the classical "secondary MR", and its prognosis is driven mainly by global LV dysfunction. Such population of patients was included in MITRA-FR trial, which showed no significant reduction in the risk of death or hospitalization from $\mathrm{HF}^{19,20}$;

2. As "disproportionate" is described the MR whose severity is far greater than anticipated, based on the LV end-diastolic volume. This type of MR could be better explained through regional LV wall motion abnormalities and asymmetrical leaflet tethering, resembling more the anatomical characteristics of "primary" MR, although it is secondary to the LV dysfunction. The COAPT trial enrolled patients with $\sim 30 \%$ greater effective regurgitant orifice area (EROA) and $\sim 30 \%$ smaller LV end-diastolic volume compared to MITRA-FR, and its outcome was positive, with a significant reduction in both morbidity and mortality (Table 1$){ }^{20}$

\section{Therapeutic strategies}

The therapeutic strategy in type IIIb MR is multidirectional, as we try to tackle the problem from multiple sides. First, we have to ensure adequate decongestion and optimization of guideline-directed medical therapy that includes angiotensin-converting enzyme inhibitors (ACE-Is) or angiotensin receptor blockers (ARBs), $\beta$-blockers, and mineralocorticoid receptor blockers. Additionally, Kang et al. recently showed that in patients with reduced left ventricular ejection fraction (LVEF) (from 25\% to 50\%) and secondary MR, the EROA was significantly more reduced in patients treated with maximal tolerable doses of sacubitril/valsartan as compared to patients treated with valsartan (absolute EROA reduction $-0.058 \mathrm{~cm}^{2}$ compared to $-0.018 \mathrm{~cm}^{2}$; relative reduction $30 \%$ compared to $9 \%$ ), after 1 year of follow-up. ${ }^{21}$ This effect can be attributed to a number of parameters such as a reduction in both afterload and preload, a more pronounced reduction in LV end-diastolic dimensions, a reduction in atrial pressures and an improvement in closing forces. ${ }^{1}$

Moreover, a crucial target is the reposition of the subvalvular apparatus by optimizing LV function and promoting reverse LV remodeling. First of all, this can be achieved through revascularization, which can improve ejection fraction and correct regional wall motion abnormalities. 
Thus, the advanced screening of myocardial viability through high-quality imaging assessment (echocardiogram (ECHO), positron emission tomography (PET) and CMR) could offer an additional "hidden" benefit. Secondly, in selected patients, using cardiac resynchronization therapy, we can improve LVEF, reduce LV volumes, synchronize PM motion, and - by restoring the equilibrium between closing and tethering forces - diminish the degree of MR. ${ }^{1,22}$

There are no studies, and therefore, no conclusive evidence, that mitral valve surgery with no option for revascularization provides any improvement in overall survival. ${ }^{12}$ New guidelines state that secondary MR (severe symptomatic secondary FMR characterized by $\mathrm{EROA} \geq 0.30$ and regurgitant volume $\geq 45 \mathrm{~mL}$ ) can be corrected, if the anatomy is suitable, by percutaneous edge-to-edge repair with a grade of recommendation IIa. ${ }^{22}$ The guidelines are restrictive to the COAPT-like patients, but they also provide the information about the required caution that needs to be taken in regard to the LVEF in MR patients. We should take into account global longitudinal strain and/or CMR evidence for fibrosis; these are the parameters reflecting the severity of the disease that might have an impact on the clinical outcome of the MR correction.

Therefore, the technique that should be considered is the transcatheter leaflet edge-to-edge approximation technique. This therapeutic intervention has been shown to be effective in improving symptoms and quality of life and reducing MR severity. Additionally, it may potentially offer survival benefit among patients with functional MR. The MitraClip ${ }^{\mathrm{TM}}$ (Abbott Laboratories, Menlo Park, USA) consists of a cobalt-chromium clip that has 2 arms and can effectively grasp and approximate the edges of opposing leaflet segments. ${ }^{17,18}$ It was designed after the surgical Alfieri technique and has gained the approval for use in primary type II MR and functional type I and IIIb MR. The PASCAL system (Edwards Lifesciences, Nyon, Switzerland) is using the same concept. Another type of transcatheter interventions in MR patients is percutaneous mitral annuloplasty, using devices such as Cardioband (Edwards Lifesciences) or Carillon (Cardiac Dimensions, Kirkland, USA). An emerging option for less invasive treatment of MR is percutaneous mitral valve replacement. The Tendyne prosthesis (transapical) has been approved in Europe, but it does not yet have the level of recognition that the percutaneous edge-to-edge valve repair has. ${ }^{22}$

\section{Conclusions}

In this overview, we described the mitral valve anatomy, focusing on its anatomical and functional relationships with the LV, and how an impaired coordination between the two can lead to valvular dysfunction with serious clinical consequences. The mitral valve is a complex, three-dimensional apparatus whose normal function depends on the equilibrium among different parts. The comprehension of these mechanisms offers us the capacity to implement appropriate therapeutic solutions in order to alleviate the burden of mitral valve disease.

\section{ORCID iDs}

Erwan Donal (D) https://orcid.org/0000-0002-9083-1582

Vasileios Panis (D) https://orcid.org/0000-0002-3296-7107

\section{References}

1. Levine RA, Hagege AA, Judge DP, et al. Mitral valve disease: Morphology and mechanisms. Nat Rev Cardiol. 2015;12(12):689-710. doi:10. 1038/nrcardio.2015.161

2. Sweeney J, Dutta T, Sharma M, et al. Variations in mitral valve leaflet and scallop anatomy on 3-dimensional transesophageal echocardiography. J Am Soc Echocardiogr. 2021;S0894-7317(21)00598-8. doi:10.1016/j.echo.2021.07.010

3. Melillo E, Ancona F, Buzzatti N, Denti P, Agricola E. A challenging mitral valve anatomy for percutaneous repair with MitraClip: Cleft posterior leaflet. Eur Heart J Cardiovasc Imaging. 2019;20(12):1433-1434. doi:10.1093/ehjci/jez175

4. Marsit O, Clavel MA, Cote-Laroche $C$, et al. Attenuated mitral leaflet enlargement contributes to functional mitral regurgitation after myocardial infarction. J Am Coll Cardiol. 2020;75(4):395-405. doi:10. 1016/j.jacc.2019.11.039

5. Dal-Bianco JP, Aikawa E, Bischoff J, et al. Myocardial infarction alters adaptation of the tethered mitral valve. J Am Coll Cardiol. 2016;67(3): 275-287. doi:10.1016/j.jacc.2015.10.092

6. Obadia JF, Casali C, Chassignolle JF, Janier M. Mitral subvalvular apparatus: Different functions of primary and secondary chordae. Circulation. 1997;96(9):3124-3128. doi:10.1161/01.cir.96.9.3124

7. Klues HG, Roberts WC, Maron BJ. Morphological determinants of echocardiographic patterns of mitral valve systolic anterior motion in obstructive hypertrophic cardiomyopathy. Circulation. 1993;87(5): 1570-1579. doi:10.1161/01.cir.87.5.1570

8. Levine RA, Vlahakes GJ, Lefebvre X, et al. Papillary muscle displacement causes systolic anterior motion of the mitral valve: Experimental validation and insights into the mechanism of subaortic obstruction. Circulation. 1995;91(4):1189-1195. doi:10.1161/01.cir.91.4.1189

9. Hung J, Otsuji Y, Handschumacher MD, Schwammenthal E, Levine RA. Mechanism of dynamic regurgitant orifice area variation in functional mitral regurgitation: Physiologic insights from the proximal flow convergence technique. J Am Coll Cardiol. 1999;33(2):538-545. doi:10.1016/s0735-1097(98)00570-1

10. Otsuji $Y$, Handschumacher MD, Schwammenthal E, et al. Insights from three-dimensional echocardiography into the mechanism of functional mitral regurgitation: Direct in vivo demonstration of altered leaflet tethering geometry. Circulation. 1997;96(6):1999-2008. doi:10.1161/01.cir.96.6.1999

11. Carpentier A. Cardiac valve surgery: The 'French correction'. J Thorac Cardiovasc Surg. 1983;86(3):323-337. PMID:6887954

12. Baumgartner H, Falk V, Bax JJ, et al. 2017 ESC/EACTS Guidelines for the management of valvular heart disease. Eur Heart J. 2017;38(36): 2739-2791. doi:10.1093/eurheartj/ehx391

13. He S, Fontaine AA, Schwammenthal E, Yoganathan AP, Levine RA. Integrated mechanism for functional mitral regurgitation. Leaflet restriction versus coapting force: In vitro studies. Circulation. 1997; 96(6):1826-1834. doi:10.1161/01.cir.96.6.1826

14. Deferm S, Bertrand PB, Verbrugge $\mathrm{FH}$, et al. Atrial functional mitral regurgitation: JACC review topic of the week. J Am Coll Cardiol. 2019; 73(19):2465-2476. doi:10.1016/j.jacc.2019.02.061

15. Deferm S, Bertrand PB, Verhaert D, et al. Mitral annular dynamics in AF versus sinus rhythm: Novel insights into the mechanism of AFMR. JACC Cardiovasc Imaging. 2021;S1936-878X(21)00440-X. doi:10.1016/j. jcmg.2021.05.019

16. Gertz ZM, Raina A, Saghy $L$, et al. Evidence of atrial functional mitral regurgitation due to atrial fibrillation: Reversal with arrhythmia control. J Am Coll Cardiol. 2011;58(14):1474-1481. doi:10.1016/j.jacc. 2011.06.032 
17. Obadia JF, Messika-Zeitoun $D$, Leurent $G$, et al. Percutaneous repair or medical treatment for secondary mitral regurgitation. $N$ Engl J Med. 2018;379(24):2297-2306. doi:10.1056/NEJMoa1805374

18. Stone GW, Lindenfeld J, Abraham WT, et al. Transcatheter mitralvalve repair in patients with heart failure. NEng/ JMed. 2018;379(24) 2307-2318. doi:10.1056/NEJMoa1806640

19. Packer M, Grayburn PA. New evidence supporting a novel conceptual framework for distinguishing proportionate and disproportionate functional mitral regurgitation. JAMA Cardiol. 2020;5(4):469-475. doi:10.1001/jamacardio.2019.5971
20. Grayburn PA, Sannino A, Packer M. Proportionate and disproportionate functional mitral regurgitation: A new conceptual framework that reconciles the results of the MITRA-FR and COAPT trials. JACC Cardiovasc Imaging. 2019;12(2):353-362. doi:10.1016/j.jcmg.2018.11.006

21. Kang DH, Park SJ, Shin SH, et al. Angiotensin receptor neprilysin inhibitor for functional mitral regurgitation. Circulation. 2019;139(11): 1354-1365. doi:10.1161/CIRCULATIONAHA.118.037077

22. Donal E, Coisne A, Sade LE. Mitral regurgitation: Not a single disease with systematic and identic functional and haemodynamic consequences. Eur Heart J Cardiovasc Imaging. 2021;22(9):974-976. doi:10. 1093/ehjci/jeab136 